(Aus dem physiologischen Institut der Universität Strassburg i. E.)

\title{
Über den elektrischen Leitungswiderstand des tierischen Körpers.
}

Von

Dr. rer. nat. Hermann Galler.

(Mit 2 Textfiguren.)

In den letzten Jahren hat man den elektrischen Strömen, welche sich vom intakten tierischen Körper ableiten lassen, grössere Beachtung geschenkt. Seit Einthoven das Saitengalvanometer zu einem vorzüglichen Registrierinstrument gemacht hat, beschäftigen sich viele Forseher mit dem Studium der Elektrokardiogramme, während andere wieder die Aktionsströme bei der Kontraktion der willkürlichen Skelettmuskulatur zum Objekt ihrer Untersuchungen machen.

Bei diesen und ähnlichen Experimenten zeichnet man Kurven auf, aus denen man nicht ohne weiteres den elektrischen Vorgang, der sich im Innern des Körpers abspielt, entnehmen kann. Denn das Saitengalvanometer registriert zwar ziemlich treu den Strom, der sich aus dem Körper in die Saite ergiesst. Nach dem $\mathrm{Ohm}$ schen Gesetze ist aber die Stärke dieses Stromes abhängig erstens von der Spannung der Stromquelle, zweitens von dem Leitungswiderstand der Strombahn. Will man also die Spannungsschwankungen in den Entstehungsorten der Elektrizität, der Herzund Skelettmuskeln, kennen lernen - und darauf kommt es doch offenbar an -, so ist dazu die genaue Erforschung des elektrischen Leitungswiderstandes des tierischen Körpers und seiner Variabilität unter dem Einfluss verschiedener Faktoren notwendig.

Auch auf einem anderen Gebiet ist dieses Problem wichtig. Wenn man den Körper von aussen Elektrizität zuführt, wie es z. B. der Arzt zu elektrodiagnostischen Zwecken macht, so wird die Stromstärke nur dann ein getreues Abbild der angelegten 
Spannung bieten, wenn der Widerstand konstant bleibt. Anderenfalls erleidet die Stromkurve eine Verzerrung, so dass beispielsweise der vermeintlich konstante Reizstrom in Wirklichkeit eine ganz andere Form erhält. Wenn diese Deformation gross ist, worüber nichts bekannt ist, so muss man ihr natürlich Rechnung tragen.

Über den Widerstand des Gesamtkörpers existieren nun viel weniger Untersuchungen, als es die Wichtigkeit der Frage vermuten liesse. Der menschliche Körper ist freilich in dieser Hinsicht öfters untersucht worden, besonders von Klinikern. Die Ergebnisse werden in jedem ausführlicheren Werk über Elektrodiagnostik und in vielen physiologischen Hand- und Lehrbüchern angeführt; ich will deshalb nicht näher auf sie eingehen. Aber beim Menschen liegen die Verhältnisse wegen der dicker und trockenen Epidermis, deren Durchfeuchtung und Blutfülle sich überdies während des Versuches leicht ändert, ganz besonders kompliziert. Ein viel besseres Versuchsobjekt ist das meines Wissens bisher fast gar nicht untersuchte Tier, besonders der Kaltblüter mit seiner dünnen und gleichmässig durehtränkten Epidermis. Dazu kommt, dass man beim Tier durch künstliche Eingriffe, wie Tötung, Enthäutung, Erhitzung, das Problem viel intensiver verfolgen kann.' $\mathrm{Zu}$ meinen Untersuchungen, die der Anregung des Herrn Professors Gildemeister entspringen, habe ich deshalb den Frosch gewählt.

Der allgemeine Plan der Untersuchung war folgender: Es sollten dem Tier von aussen Elektroden angelegt und Ströme verschiedener Art (teils Gleich-, teils Wechselströme) durch diese hindurchgeschickt werden. Jedesmal war dann nach der für den betreffenden Fall geeignetsten Methode der Widerstand zu bestimmen. Nach den Versuchen am Menschen war vorauszusehen, dass man je nach der Grösse und dem Abstand der Elektroden verschiedene Werte erhalten werde, und dass auch noch andere Momente, wie Art des Stromes, Dauer der Durchströmung usw., sich geltend machen würden. Es kam hier nicht darauf an, absolute Widerstandswerte festzulegen, sondern es war meine Absicht, ganz allgemein die Bedingungen zu ermitteln, von denen eine Veränderung des Widerstandes abhängt.

Im weiteren Verlauf der Untersuchung zeigte es sich, dass sich im Tierkörper bei der Durchströmung recht beträchtliche ele $\mathrm{k} t \mathrm{r}$ i s c h $\mathrm{e}$ Gegenkräfte entwickeln, welche sich als "scheinbarer Widerstand " geltend machen. Auch diese Tatsache war nach den Untersuchungen 
von du Bois-Reymond, Hermann, Hering u. a. über innere Polarisation tierischer Gewebe vorauszusehen. Meine Untersuchungsmethode macht es möglich, die Grösse dieser Gegenkräfte während der Durchströmung zu messen. In dem strengen Nachweis, dass der Gleichstromwiderstand zum Teil durch entstehende Gegenkräfte vorgetäuscht wird, liegt der Sehwerpunkt der nachstehenden Arbeit.

\section{Methode.}

Da die Arbeit während des Winters (1910-1911) von November bis März ausgeführt wurde, benutzte ich dazu Winterfrösche beider hier vorkommender Arten, meistens jedoch Eskulenten von mittlerer Grösse. Die Tiere, durch Aufbinden auf das Ewald'sche Froschkreuz möglichst fixiert, fanden teils lebend, teils frisch getötet Verwendung. Bei einigen Versuchen wurden den getöteten Fröschen die Haut abgezogen; in anderen Fällen wurden die Tiere gekocht und wieder abgekühlt. Davon wird noch später die Rede sein. Es gelangten bei den Untersuchungen sowohl Gleich- wie Wechselströme zur Anwendung.

Die Gleichströme wurden von 1-6 Akkumulatoren (also 2 bis 12 Volt) geliefert. Zur Ablesung der resultierenden Stromstärke. wurde ein sehr empfindliches Milliamperemeter benutzt, welches $270 \mathrm{Ohm}$ inneren Widerstand besass und bei dem 27 . Skalenteile 1 Milliampere bedeuteten. Nach dem $0 \mathrm{hm}$ 'schen Gesetz wurde dann aus der abgelesenen Stromstärke und der angelegten Spannung der Widerstand errechnet, wobei natürlich der vorher gemessene Widerstand der Elektroden berücksichtigt wurde. Abgelesen wurden noch halbe Skalenteile.

Die Wechselströme wurden meistens geliefert von einem kleinen Induktorium mit $\mathrm{W}$ agner'schem Hammer, der ca. 120 Unterbrechungen in der Sekunde machte. Bei einzelnen Versuchen wurde die Stärke des Wechselstromes durch Verschieben der Rollen variiert. In einigen Versuchsreihen benutzte ich frequentere Wechselströme $(460-1020$ in der Sekunde). Diese wurden von einem Induktorium geliefert, in dessen primären Kreis ein Quecksilberturbinenunterbrecher eingeschaltet war. In anderen Versuchen wieder gelangte pulsierender Gleichstrom (durch den Turbinenunterbrecher 460-1020 mal in der Sekunde unterbrochen) zur Anwendung. Der Widerstand, welchen das Tier dem Wechsel- oder dem zerhackten Gleichstrom entgegen- 
setzte, konnte mit Hilfe der Kohlrausch'schen Methode (Wheats ton e' sche Brücke und Telephon) gemessen werden. Das Tonminimum war im allgemeinen recht verwaschen; doch war es oft möglich, eine Verbesserung dadurch zu erhalten, dass ein kleiner Kondensator parallel dem Stöpselrheostaten, welcher den Vergleichswiderstand bildete, eingeschaltet wurde ${ }^{1}$ ).

Ich benutzte unpolarisierbare Elektroden, um alle Fehler zu vermeiden, die von metallischer Polarisation herrühreu konnten. Das Nähere wird bei den einzelnen Versuchen erwähnt werden. Die Elektroden stellte ich so her, dass ich ca. $6 \mathrm{~cm}$ lange Glasröhrchen auf einer Seite mit Schweinsblase zuband, dann mit konzentrierter Zinksulfatlösung füllte und in die Lösung mit Hilfe eines kleinen Gummischlauches einen gut amalgamierten Zinkstab eintauchte. Die Glasröhren (3 Paare) hatten verschiedene Weite, und zwar hatten die engen einen Durchmesser von $5 \mathrm{~mm}$, die mittleren einen solchen von $8,5 \mathrm{~mm}$ und die weiten von $17 \mathrm{~mm}$. Die Flächen der aufgesetzten Elektroden betrugen daher $19,6,56,7$ und $227 \mathrm{qmm}$; sie verhielten sich also zueinander sehr nabe wie $1: 3: 12$. $\mathrm{Zu}$ den einzelnen Versuchen wurden immer 2 Elektroden von dem gleichen Durchmesser frisch mit Zinksulfatlösung gefüllt. Sie wurden durch an Stativen befestigten Klemmen gebalten und mässig stark gegen den Froschkörpe ${ }^{r}$ gedrückt. Bei den jeweiligen Untersuchungen auf ihre Unpolarisierbarkeit erwiesen sich diese Elektroden insofern als gut, als ihr Gleich- und Wechselstromwiderstand immer fast von derselben Grösse war.

Während ich bei vielen Versuchen nur Gleichstrom, bei anderen nur Wechselstrom verwendete, nahm ich einige Male die Messungen der beiden Widerstände ganz kurz hintereinander an demselben Objekt vor, und zwar mit Hilfe einer Umschaltevorrichtung, welche ein rasches Vertauschen der einen mit der anderen Anordnung ermöglichte. Ferner erwies es sich im Verlaufe der Untersuchungen als notwendig, die Messung mit Gleich- und Wechselstrom am selben Objekt zu gleicher Zeit vorzunehmen. Hierzu war wiederum eine besondere Schaltung nötig, die später beschrieben werden soll.

1) Herr Professor Gildemeister hat, wie er mir mitteilt, in neuester Zeit festgestellt, dass die so gefundeuen Widerstandswerte wegen der Polarisation unter Umständen beträchtlich von den wahren abweichen. Die weiter unten mitgeteilten Zahlenwerte sind daher nur als angenähert zu betrachten. 
Das Zimmer, in welchem die Untersuchungen vor sich gingen und die Frösche aufbewahrt wurden, hatte eine Temperatur von etwa $18^{\circ} \mathrm{C}$. Doch wurden auch : Frösche direkt aus dem kühlen Keller (etwa $7^{\circ}$ ) geholt und sofort untersucht. Ein qualitativer Unterschied im Resultat ergab sich aber aus der Versehiedenheit der Temperaturen nicht; inwiefern etwa die absoluten Werte dadurch verändert wurden, habe ich nicht weiter untersucht.

\section{Gleichstromversuche.}

Die hierzu benutzte Schaltung war sehr einfach: die nötigen Elemente, ein Stromwender, ein Schlüssel, das Milliamperemeter und die Elektroden waren in einen Stromkreis eingeschaltet. Das Milliamperemeter war fast aperiodisch und sehr schnell in seiner Einstellung, so dass schon in einer Sekunde abgelesen werden konnte.

Wurde jetzt ein Frosch zwischen die Elektroden gebracht und der Schlüssel geschlossen, so erreichte das Instrument bei nicht zu grossen Stromstärken sehr schnell (in wenigen Sekunden) einen festen Stand. Auf diesem verharrte es ziemlich ruhig längere Zeit (bis zu 10 Minuten beobachtet). Dies muss erwähnt werden, da es eine bekannte Tatsache ist, dass beim Durchleiten des elektrischen Stromes durch den Menschen die Stromstärke sich nach der Einschaltung längere Zeit langsam ändert.

Benutzt man mehrere Elemente ( 6 Volt und mehr), so sinkt die Stromstärke allmählich ein wenig. Wendet man jetzt den Strom, so steigt die Intensität ziemlich rasch auf ihren früheren Wert und darüber hinaus, um bald wieder zu sinken. Dies kann man beliebig oft wiederholen.

Aus der Stromstärke wurde der scheinbare Widerstand ${ }^{1}$ ) des Froschkörpers folgendermaassen gefunden: Man berechnet zuerst aus ihr und der angelegten Spannung nach dem $\mathrm{Ohm}$ 'schen Gesetz den Widerstand der gesamten Anordnung und zieht davon den Widerstand der Elektroden und des Milliamperemeters ab. Der Widerstand der Elektroden wurde vor jedem Versuch neu bestimmt; er betrug bei den grossen ca. $200 \mathrm{Ohm}$, bei den mittleren ca. 400 und bei den kleinen ca. $600 \mathrm{Ohm}$. Der Widerstand des Milliamperemeters betrug $270 \mathrm{Ohm}$.

1) Warum hier von dem "scheinbaren" Widerstand die Rede ist, ergibt sich aus den folgenden Abschnitten. 
Es soll hier gleich bemerkt werden, dass es gleicbgültig ist, ob der Frosch lebend oder aber frisch getötet ist. Auf den Widerstand (sowohl für Gleich- als für Wechselstrom) hat die Intaktheit des Zentralner vensystems keinen Einfluss. Im übrigen ist von dem scheinbaren Widerstand zu sagen, dass er bei dem unversehrten Tier abhängt: 1. von der Grösse der Elektroden und ihrem Abstand voneinander; 2. von der angelegten Spannung, d. h. also von der Stromstärke. Der Einfluss der einzelnen Faktoren soll in folgendem kurz beschrieben werden. Da die durchströmten Gewehe nicht homogen sind, und da auch beträchtliche individuelle Unterschiede vorkommen, führe ich nur einzelne Beispiele an, die das Typische erkennen lassen.

Grösse der Elektroden. Der Widerstand ${ }^{1}$ ) ist naturgemäss desto kleiner, je grösser die Elektroden. Jedoch sinkt er nicht im gleichen Verhältnis, wie die Elektrodenoberfläche steigt. Beispielsweise betrug er unter sonst ganz gleichen Bedingungen (abgerundet) :

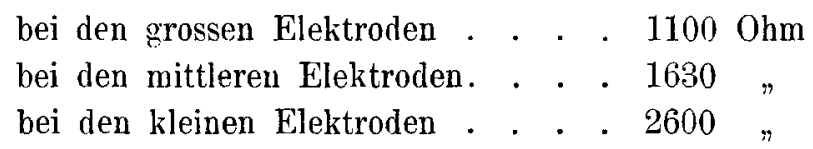

Diese Werte ergaben sich als Mittelwerte aus mehreren Versuchen unter gleichen Bedingungen (gleicher Abstand). Aus ihnen ist ersichtlich, dass die scheinbaren Widerstände sich nicht umgekehrt wie die Elektrodenflächen $(1: 3: 12)$, sondern eher wie die Elektrodendurchmesser $(1: 1,7: 3,4)$ oder noch besser wie die dritten Wurzeln aus den Elektrodenflächen $(1: 1,44: 2,29)$ verhalten.

Abstand der Elektroden. Der scheinbare Widerstand wird mit Zunahme des Abstandes der Elektroden grösser; jedoch erfolgt die Zunahme des Widerstandes weniger schnell als die $\mathrm{Zu}$ nahme des Abstandes (gemessen von Mitte zu Mitte der Elektrodenfläcben). Am besten kann dies durch folgenden Versuch erläutert werden: Verwendet wurde ein mittelgrosser lebender Frosch (Esculenta) mit intakter Haut, welcher mehrere Tage vorher im Zimmer bei $18^{\circ}$ aufbewahrt war. Die mittelgrossen $(8,5 \mathrm{~mm}$ Durchmesser) Elektroden hatten einen Widerstand von $430 \mathrm{Obm}$.

Wie aus der Tabelle (s. S. 162) ersichtlich ist, hat sich der scheinbare Widerstand nur etwa verdoppelt, während der Elektrodenabstand beinahe auf das Zwanzigfache gestiegen ist.

1) Wenn keine andere Angabe gemacht ist, dienteu zur Messung immer 2 Volt. P flüger's Arehiv für Physiologie. Ba. 149. 


\begin{tabular}{|c|c|c|}
\hline $\begin{array}{l}\text { Elektroden- } \\
\text { abstand } \\
\text { mm }\end{array}$ & $\begin{array}{c}\text { Scheinbarer } \\
\text { Widerstand } \\
\text { Ohm }\end{array}$ & Körperregionen \\
\hline $\begin{array}{r}3 \\
3 \\
25 \\
25 \\
50\end{array}$ & $\begin{array}{l}1620 \\
1780 \\
2270 \\
2500 \\
3830\end{array}$ & $\begin{array}{l}\text { quer durch den lirken Oberschenkel } \\
\text { quer durch den rechten Oberschenkel } \\
\text { obere Bauchgegend } \\
\text { Rücken } \\
\text { Bauch und rechter Oberschenkel }\end{array}$ \\
\hline 50 & 8830 & $\begin{array}{l}\text { Eine Elektrode auf den Bauch, die } \\
\text { andere auf den linken Oberschenkel }\end{array}$ \\
\hline $\begin{array}{l}55 \\
55\end{array}$ & $\begin{array}{l}3480 \\
3480\end{array}$ & $\begin{array}{l}\text { Rücken und linker Obersubenkel } \\
\text { Rücken und rechter Oberschenkel }\end{array}$ \\
\hline
\end{tabular}

Die Elektroden setzte ich mit Ausnahme der beiden ersten Versuche (quer durch die Oberschenkel) einander parallel, d. h. beide vertikal auf, so dass also die Stromlinien, die aus der einen Elektrodenfläche senkrecht herauskamen, sich krümmen mussten, um in die andere Elektrode ebenso eindringen zu können. Die Länge der Stromfäden ist also grösser als die in der Tabelle angegebenen Beträge des Abstandes der Elektroden.

Die zuletzt angeführten Versuche machten es wahrscheinlich, dass der grösste Teil des scheinbaren Widerstandes in der Haut seinen Sitz hatte. Denn in diesem Fall konnte ja der Widerstand bei der Vergrösserung des Elektrodenabstandes nur wenig steigen. Bei kleinem Abstand setzte sich nämlich die Strombahn zusammen aus Haut - kurzem Körperweg - Haut, bei grossem Abstand aus Haut - langem Körperweg - Haut; es kam also im zweiten Falle nur ein relativ kleiner Summand (Differenz langer Körperweg kurzer Körperweg) dazu. Um diese Folgerung zu prüfen, machte ich nun Experimente an enthäuteten Fröschen.

Entbäutung. Die Versuche wurden in rer Weise vorgenommen, dass man zuerst bei unversehrten Fröschen den Widerstand maass, dann die Frösche tötete, die Haut abzog und nun den Widerstand an den enthäuteten Tieren wieder feststelite. Nachher wurde öfters zur Kontrolle die abgezogene Haut wieder aufgelegt und die Messung wiederholt. Die Elektroden wurden in gleichem Abstand wie bei den lebenden Tieren aufgesetzt. Es zeigte sich dann, dass bei sonst ganz gleichen Versuchsbedingungen der Widerstand in den enthäuteten Tieren sich wesentlich verminderte. Von verschiedenen hierüber angestellten Versuchen sei nur einer angeführt:

Die mittelgrossen Elektroden (8,5 $\mathrm{mm}$ Durchmesser) wurden in einem Abstand von $25 \mathrm{~mm}$ auf den Bauch eines Frosches gesetzt. 
Es ergab sich nun bei dem intakten Tier ein Widerstand von $2500 \mathrm{Ohm}$; als dasselbe getötet und enthäutet war, nur noch ein solcher von $1330 \mathrm{Ohm}$.

Die Haut allein für sich doppelt gefaltet ergab mit denselben Elektroden einen Widerstand von $1620 \mathrm{Ohm}$.

Andere Versuche ergaben ähnliche Werte. Also hat tatsächlich ein sehr grosser Teil des Widerstandes in der Haut seinen Sitz.

Erhitzung. $\mathrm{Zu}$ diesen Versuchen wurden die frisch getöteten Tiere kurze Zeit in kochendes Wasser gelegt, wieder abgekühlt und dann der Widerstand gemessen. Gegenüber den lebenden oder nicht erhitzten toten Tieren war der Widerstand bedeutend herabgesetzt

Beispielsweise war er bei einem Versuch von $1670 \mathrm{Ohm}$ auf $5200 \mathrm{hm}$ vermindert.

Variierung der angelegten Spannung (und damit der Stromstärke). Bei allen bisherigen Untersuchungen wurde nur ein einziger Akkumulator von 2 Volt Spannung benützt. Bei den Versuchen, die jetzt beschrieben werden sollen, wurde durch Hintereinanderschaltung mehrerer Akkumulatoren die Spannung erhöht, und zwar auf 4, 6, 8, 10 und 12 Volt. In mehreren anderen Versuchsreihen kamen auch niedrigere Spannungen von $0,2-2$ Volt zur Anwendung. Eine höhere Steigerung der Spannung als 12 Volt war nicht möglich, weil dann mein Milliamperemeter nicht mehr ausreichte.

Es ergab sich, dass bei Steigerung der Spannung, womit natürlich unter sonst gleichen Bedingungen auch die Stromstärke wächst, der scheinbare Widerstand sank; so z. B. hetrug dieser in einem Falle:

Esculenta, 18 ${ }^{\circ}$ mittlere Elektroden, $25 \mathrm{~mm}$ Abstand:

\begin{tabular}{|c|c|c|c|c|c|}
\hline ei 2 & Volt & 2530 & Ohm & $\left(\mathrm{St}_{1}\right.$ & \\
\hline$n$ & n & 2050 & $\eta$ & ( & 1,33 \\
\hline$"$ & " & 1940 & $n$ & $"$ & 2,07 \\
\hline 8 & $n$ & 1820 & $n$ & n & 2,84 \\
\hline
\end{tabular}

Wenn man nun wieder rückwärts geht, von höheren zu niedrigeren Spannungen, so findet man nicht die auf dem Hinwege ermittelten Widerstandswerte wieder, sondern der scheinbare Widerstand ist auf dem Rückwege bei gleicher Spannung niedriger als auf dem Hinwege. Das ist an folgendem Beispiele zu erkennen: 


$\begin{array}{lcccc}\text { Spannung Volt: } & 0,2 & 0,6 & 1 & 2 \\ \text { Widerstand Ohm: } & 6000 \rightarrow 4040 \rightarrow 3745 \rightarrow 3330 \\ 3745 & \leftarrow 3480 \leftarrow 3430\end{array}$

Man kann also, um einen Ausdruck der Physiker zu brauchen, sagen, dass der scheinbare Widerstand von der Vorgeschichte abhängig ist: er ist niedriger, wenn bei dem vorhergehenden Versuch stärkere Ströme zur Anwendung gekommen sind. Diese Depression verschwindet wieder, wenn man das Versuchsobjekt einige Zeit in Ruhe lässt.

Schickt man durch ein System, das wie der tierische Körper Elektrolyte und balbdurchlässige Membranen enthält, einen Gleichstrom, so müssen nach den heutigen Anschauungen der physikalischen Chemie in ihm Veränderungen eintreten, die sich als elektromotorische Gegenkräfte geltend machen. Misst man nun, wie ich es in den bisher mitgeteilten Versuchen gemacht habe, die angelegte Spannung $e$ und die Stromstärke $i$ und berechnet daraus nach dem $\mathrm{Ohm}$ 'schen Gesetze $w=\frac{e}{i}$ den Widerstand $w$, so muss man für diesen, sofern die Gegenkräfte nicht berücksichtigt werden, falsche, und zwar zu hohe, Beträge erbalten, da ja in Wirklichkeit die maassgebende Spannung im Zähler des Bruches nicht den Wert $e$, sondern den kleineren $e-\varepsilon$ besitzt ( $\varepsilon=$ elektromotorische Gegenkraft).

Dasselbe gilt, wenn der Gleichstromwiderstand nach irgendeiner anderen Methode, z. B. nach Wheatstone, gemessen wird.

Die bisher ermittelten Werte waren also höcbstwahrscheinlich nicht wirkliche, sondern nur scheinbare Widerstände.

Um die besagte Schwierigkeit zu vermeiden, verwendete ich jetzt Wechselströme, wie es ja allgemein bei der Messung von Elektrolyten äblich ist.

\section{Widerstandsmessnng mit Wechselströmen.}

Die Methode war die bekannte Kohlrausch'sche Brückenmethode mit einem Telephon als Nullinstrument. Die Wechselströme wurden von einem kleinen Induktorium geliefert, dessen primärer Strom teils durch einen W ag n er'schen Hammer (Frequenz etwa 120), teils durch eine Quecksilberturbine (Frequenz 480-1020 pro Sek.) unterbrochen wurde. Auch hier wurden wieder Grösse und Abstand der Elektroden, ferner Stärke und Frequenz der Mess- 
ströme und schliesslich der Zustand des Tieres (lebend oder getötet, intakt oder enthäutet) variiert. Wie schon oben erwähnt, hat die blosse Abtötung durch Zerstörung des Zentralnervensystems keinen Einfluss auf die Messungsresultate. Die Ergebnisse waren folgende:

Grösse der Elektroden. Hier kamen ebenfalls die vorher beschriebenen Elektrodenpaare von 19,6, 56,7 und $227 \mathrm{qmm}$ Fläche zur Anwendung.

Auch bei dieser Methode zeigte sich, dass der Widerstand mit Zunahme der Elektrodengrösse sank. Er betrug beispielsweise in einem Falle bei den kleinen Elektroden $1500 \mathrm{Ohm}$, bei den mittleren 1050 , bei den grossen $670 \mathrm{Ohm}$, in einem anderen, bei grösserem Abstand, 3600, 1770 und $1280 \mathrm{Ohm}$.

Im allgemeinen zeigte es sich auch hier, dass das Sinken des Widerstandes nicht in Proportion mit dem Ansteigen der Flächengrösse der Elektroden ist.

Abstand der Elektroden. Je grösser der Abstand der Elektroden voneinander, desto mehr steigt auch der Widerstand an. Folgendes Beispiel sei angeführt:

Esculenta bei $18^{\circ}$ C. aufbewahrt. Mittlere Elektroden von $8,5 \mathrm{~mm}$ Durchmesser, die einen Widerstand von $300 \mathrm{Ohm}$ haben.

\begin{tabular}{c|c|c}
\hline $\begin{array}{c}\text { Elektroden- } \\
\text { abstand } \\
\text { mm }\end{array}$ & $\begin{array}{c}\text { Widerstand } \\
\text { Ohm }\end{array}$ & Körperregionen \\
\hline 5 & 700 & Quer durch den Bauch, zusammengedrückt \\
10 & 850 & Quer durch den Bauch \\
36 & 1200 & Bauch \\
65 & 2000 & Bauch oben und rechter Oberschenkel
\end{tabular}

Auch hier wächst der Widerstand nicht so schnell wie der Abstand der Elektroden.

Enthäutung. Wie beim Gleichstrom sinkt auch beim Wechselstrom in diesem Fall der Widerstand eines entbäuteten Tieres; jedoch ist die Abnahme kleiner als im ersteren Falle und beträgt bei den mittelgrossen Elektroden nur etwa 200-300 Ohm, während sie bei Gleichstrom unter denselben Bedingungen etwa $1000 \mathrm{Ohm}$ betrug. Man kann sagen, dass der Wechselstromwiderstand des Gesamtkörpers nur in geringem Maasse in der Haut lokalisiert ist.

Erhitzung. Bei gekochten Tieren sinkt (nach der Abkühlung gemessen) der Widerstand beträchtlich, etwa bis auf die Hälfte. 
Einfluss der Stärke und Frequenz der Messströme. Die Stärke des Weelsselstromes wurde, freilich nur in mässigen Grenzen, durch verschiedene Rollenabstände ${ }^{1}$ ), die Frequenz (120 bis 1020) durch verschiedene Unterbrecher variiert. Aber beide Veränderungen brachten keinen merklichen Einfluss auf den Widerstand hervor. Das Minimum der Lautstärke blieb auf der Messbrücke immer merklich auf derselben Stelle. Jedoch ist es möglich, dass mir kleine Verschiebungen wegen der Breite des Minimums entgangen sind.

Vergleicht man die zuletzt mitgeteilten Versuche mit den vorherigen, mit Gleichstrom angestellten, so sieht man zwei wesentliche Unterschiede. Bei Gleichstrom ändert sich der Widerstand, wenn die angelegte Spannung und damit die Stromintensität variiert wird, und er hat zu einem sehr grossen Teil in der Haut seinen Sitz. Bei Wechselstrom kommt es in den Grenzen meiner Versuche auf die Intensität (und die Frequenz) der Messströme nicht merklich an, und die Haut setzt diesen einen im Verhältnis zu ihrer Dicke recht beträchtlichen, aber doch nicht. so auffälligen Widerstand entgegen.

Die nun mitzuteilende Versuchsreihe bezieht sich auf den Vergleich des Wechselstrom- und des Gleichstromwiderstandes unter gleichen Versuchsbedingungen.

Jetzt wurde eine Umschaltevorrichtung (Wippe ohne Kreuz) angebracht, mittels deren den Elektroden und damit dem Tiere nach Belieben die eine oder die andere Stromart zugeleitet werden konnte.

Durch viele Versuche wurde festgestellt, dass bei dem unversehrten Frosch unter vollständig gleichen Versuchsbedingungen der Gleichstromwiderstand bei einer Stromspannung von 2 Volt durchschnittlich (in roher Annäherung) etwa doppelt so gross ist als der Wechselstromwiderstand. Das ändert sich aber sehr wesentlich, wenn das Tier gekocht und wieder abgekühlt wird. Beide Stromarten finden dann gleichen, und zwar stark verminderten Widerstand. Das vorher verwaschene Tonminimum wird dann ganz scharf.

Von den vielen Versuchen sei hier nur ein Beispiel angeführt:

Grosse Elektroden (D. 17,5 mm). Abstand: $25 \mathrm{~mm}$.

Froseh lebend. Gleichstromwiderstand: $1100 \mathrm{Ohm}$.

" "Wechselstromwiderstand : 614 "

1) Es kam niemals zu einer sichtbaren Muskelkontraktion durch die frequenten Reize. 
Nach Kochen und Abkühlung war der erste Widerstand auf 280, der zweite auf 284 Ohm gesunken.

Enthäutet man den Frosch, so sind auch ohne Erhitzung, wie aus dem Vorhergehenden schon gefolgert werden kann, die beiden Widerstände nicht so sehr verschieden wie bei dem intakten Tier.

Die Ergebnisse des letzten Abschnittes sprechen sehr für die Richtigkeit der 'oben geäusserten Auffassung, dass der hohe Gleichstromwiderstand durch im Gewebe entstehende elektromotorische Gegenkräfte vorgetäuscht wird, während der wirkliche $0 \mathrm{hm}$ 'sche Widerstand viel niedriger ist. Wenn diese bei 2 Volt angelegter Spannung 1 Volt betrügen, so bliebe als wirksame Spannung nur noch 1 Volt übrig; der resultierende Strom hätte nur die halbe Stärke und man würde daraus den doppelten Widerstand berechnen, welcher Wert tatsächlich für Gleichstrom gefunden wird.

Jedoch können die Verhältnisse auch anders liegen. Es wird ja durch den Gleichstrom die Konzentration der Elektrolyte an einzelnen Stellen vermehrt, an anderen aber vermindert. Die letztere Veränderung könnte so weit gehen, dass dort schlecht leitende Schichten entstehen. Nach dieser Auffassung wäre aiso die Erhöhung des Gleichstromwiderstandes teilweise oder ganz wirklich, nicht nur scheinbar.

Zwischen diesen beiden Möglichkeiten kann ein einfacher Versuch entscheiden. Man braucht nur, während der Gleichstrom hindurchfliesst, den Wechselstromwiderstand zu bestimmen und festzustellen, ob letzterer erböht ist oder nicht. Im positiven Falle hat der Gleichstrom wirklich den $0 \mathrm{hm}$ 'schen Widerstand verändert; im negativen handelt es sich ausschliesslich um elektromotorische Gegenkräfte ${ }^{1}$ ).

Die Anordnung dieser Versuche und ihre Resultate sind im nächsten Abschnitt beschrieben.

1) Als Herr Dr. Galler mit mir diesen Versuchsplan entwarf, hatten wir noch keine Kenntnis daron, dass vor kurzem Aebly unter der Leitung von Zang ger (Zur Analyse der physikalischen Vorbedingungen des psychogalvanischen Reflexes bei exosomatischer Stromquelle. Inaug. - Diss. Zürich 1910) eine verwandte Frage mit naturgemäss ganz ähnlicher Methodik am Menschen bearbeitet hat. Er findet, dass ein sehr schwacher Gleichstrom den Wechselstromwiderstand, von Hand zu Hand gemessen, nicht verändert, und zieht daraus auch den Schluss, dass ausschliesslich elektromotorische Gegenkräfte auftreten. Gildemeister. 


\section{Gleichzeitige Widerstandsmessung mit Gleich- und Wechselstrom.}

Die Schaltung ist aus Fig. 1 zu ersehen. Sie ist ganz einfach: In denjenigen Zweig der Wheatstone'schen Brücke, die wie gewöhnlich das zu messende Objekt $F r$ (Elektroden + Tier) enthält (Fig. 1 rechts oben), ist ausserdem noch eine Stromquelle $E$ und das Gleichstrommessinstrument $M A$ eingeschaltet. Man kann nun in gewöhnlicher Weise den $W$ echselstromwiderstand von $E+M A+F r$ mittels des Telephons messen. Dass dabei das letztere von Gleichstrom durchflossen wird, beeinflusst höchstens ein wenig seine Empfindlichkeit. Den Widerstand von $F r$ findet man dann durch Subtraktion der bekannten Grössen $E$ (praktisch gleich Null) und $M A$ (270 Ohm). Störende Selbstinduktion oder Kapazität war hier-nicht vorhanden.

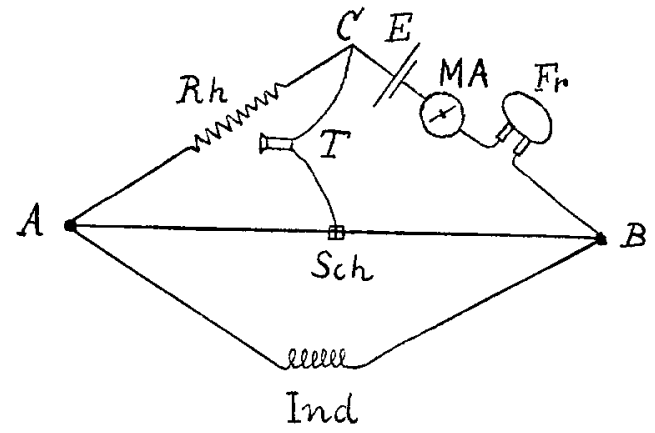

Fig. 1. $A B$ Messdraht, Sch Schieber, Ind Induk- strom durchflossen wird, torium, $R h$ Rheostat, $T$ Telephon, $E$ Element, $M M A$ Milliamperemeter, $F r$ Frosch mit Elektroden. widerstand ist in bekannter Weise den Angaben des Instrumentes $M A$ zu entnehmen, das die im rechten oberen Zweige herrschende Intensität des Dauerstromes angibt. Dass es ausserdem noch von Wechseltut nichts zur Sache. Bei

Der Gleichstromder Verwertung seiner Angaben ist $\mathrm{zu}$ berücksichtigen, dass der Gleichstromkreis mehrere Abzweigungen besitzt. So ist $B \operatorname{Ind} A$ eine Nebenschliessung zu $A B$, ebenso $S c h A R h C$ eine solche zu $S c h T C$. Da aber $A B$ und $T$ in den maassgebenden Versuchen sehr kleine Widerstandswerte im Verhältnis $\mathrm{zu}$ ihren Nebenschliessungen besassen $(A B 7 \mathrm{Obm}, T 32 \mathrm{Ohm})$, so brauchen die viel schlechter leitenden Nebenschliessungen nicht in Rechnung gezogen zu werden. Die Anwendung der bekannten Sätze über Stromverzweigung lehrt, dass man einen Fehler von nur wenigen Ohm begeht, wenn man nur den Stromverlauf in $E M A F r B S c h T C$ ins Auge fasst und demzufolge von den Angaben von $M A$ den Widerstand des Telephons $T$, des Instrumentes $M A$ und des halben Messdrahtes $S c h B$ abzieht, um den Gleichstromwiderstand von $F r$ zu finden.

Auf diese Weise konnten gleichzeitig die beiden uns interessierenden Werte ermittelt werden. Schon die ersten Versuche 
zeigten mir; dass die Unterschiede zwischen ihnen ebenso gross blieben wie bei getrennter Messung, d. h. also, dass der Gleichstrom den Wechselstromwiderstand nicht erhöhte.

Nun wurde die Schaltung noch etwas verändert, so dass der Wechselstromwiderstand mit und ohne Gleichstrom, der Gleichstromwiderstand mit und ohne Wechselstrom gemessen werden konnte. Erst diese Anordnung lieferte die definitive Entscheidung der aufgeworfenen Frage.

Zwischen Induktorium und Messdraht wurde ein Kurzschlussschlüssel $K$ (Fig. 2) eingefügt. War er geöffnet, so gingen die Wechselströme ungehindert in die Brücke; andernfalls wurden sie abgeblendet. $\mathrm{Ob}$ sie den Froseh durehflossen oder nicht, sein Gleichstromwiderstand blieb ungeändert.

Ferner kam an die Stelle von $E$ eine Wippe ohne Kreuz $W$, die auf der einen Seite zu den Elementen führte, während die andere Seite durch einen Draht kurzgeschlossen war (s. Fig. 2). Je nach ihrer Lage schaltete sie die Stromquelle ein oder aus.

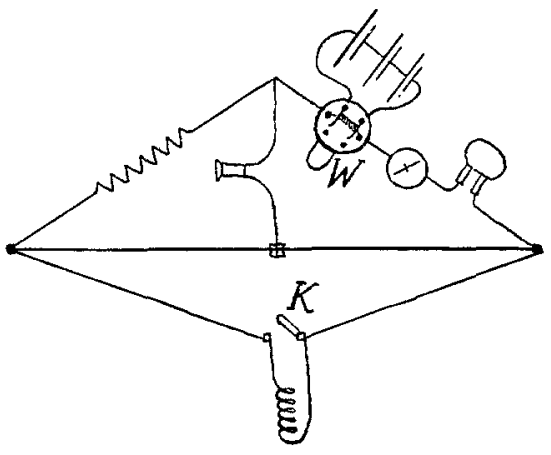

Fig. 2.

Der maassgebende Versuch verlief jetzt in folgender Weise: Die Wippe $W$ wurde zuerst nach unten umgelegt, so dass das Präparat keinen Gleichstrom bekam. Mittels des Telephons wurde der Wechselstromwiderstand bestimmt; der Rheostat wurde dabei so gestöpselt, dass das Minimum auf die Mitte des Messdrahtes zu liegen kam. Jetzt wurde die Wippe rasch umgelegt und nachgesehen, ob durch den nan fliessenden Gleichstrom das Minimum eine andere Lage bekam.

Was die Ergebnisse dieser Versuche anbetrifft, so sind sie ganz eindeutig, sofern man die Wechselstrommessungen s of ort nach dem Einschalten des Gleichstroms anstellt. Das Minimum blieb dann stets an derselben Stelle, d. h. der Wechselstromwiderstand wird vom Gleichstrom nicht beeinflusst.

Zur Erläuterung möge folgender Versuch dienen:

Kleine Esculenta, ziemlich mager. Mittelgrosse Elektroden mit $25 \mathrm{~mm}$ Abstand auf den Bauch gesetzt. 
$\begin{array}{llllllll}\text { Benutzte Gleichstromspannung in Volt } & 2 & 4 & 6 & 8 & 10 & 12\end{array}$ Wechselstromwiderstand vor Einschal-

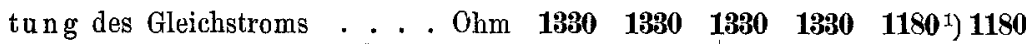

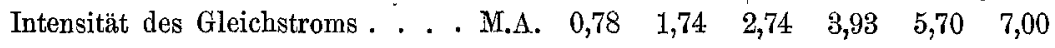

Daraus berechneter Gleichstromwiderstand

Ohm $2010 \quad 1740 \quad 1630 \quad 1480 \quad 1330 \quad 1300$

Wechselstromwiderstand während des

Gleichstroms . . . . . . . . Ohm $13301330 \quad 1330 \quad 1330 \quad 1180 \quad 1180$

Die beiden fettgedruckten Zeilen sind.ganz identisch.

Man kann also sagen: Während seines Fliessens beeinflusst der Gleichstrom den Widerstand seiner Bahn nicht, sondern dieser behält en niedrigen Wert, welcher dureh Wechselstrommessungen gefunden wird. Die Differenz ist ausschliesslich auf elektromotorische Gegenkräfte zu beziehen.

Nach Verlauf einiger Sekunden ändern sich die Verhältnisse, wenn der Gleichstrom stark ist (Spannung 6 Volt oder mehr). Dann beginnt der Wechselstromwiderstand langsam zu steigen, während der Zeiger des Milliamperemeters durch sein Sinken auch die gleichzeitige Erhöhung des Gleichstromwiderstandes (schon S. 160 erwähnt) anzeigt. Bei nunmehriger Stromwendung kehrt sich alles um: Wechselstrom- und Gleichstromwiderstand sinken rasch, oft bis unter ihren ursprünglichen Wert, um bald wieder langsam zu steigen.

Diesen Erscheinungskomplex wird man wohl sekundären Umständen, wie sie oben als möglich hingestellt sind (Bildung besonders gut- oder schlechtleitender Schichten, Veränderung der Membranen u. dergl.), zuschreiben müssen.

Zusammenfassend kann ich sagen: Der Gleicbstrom weckt zuerst nur elektromotorische Gegenkräfte; erst sekundär treten dazu, wenn er die genügende Stärke hat, Variationen des wahren Widerstandes.

\section{Versuche mit pulsierendem Gleichstrom.}

Um meine Ergebnisse von einer anderen Seite aus zu prüfen, leitete ich dem Messdraht der Whe atstone'schen Brücke, nachdem das Induktorium entfernt war, pulsierenden Gleichstrom (durch einen Turbinenunterbrecher $1020 \mathrm{mal}$ in der Sekunde unterbrochen)

1) Die Elektroden sind ein wenig verschoben. 
zu. Im übrigen blieb die Schaltung so, wie sie in Fig. 2 skizziert ist; die Wippe $W$ lag nach unten.

Der unterbrochene Gleichstrom kann aufgefasst werden als ein Wechselstrom, auf den sich ein stetiger Gleichstrom superponiert. Nach meinen früheren Ergebnissen musste ich erwarten, dass das telephonische Minimum seine frühere Lage, die vorher mit Induktionsströmen bestimmt war, behalten würde. In der Tat bestätigte der Versuch meine Erwartungen vollkommen.

\section{Über die Grösse und das Wesen der elektromotorischen Gegenkräfte.}

Es ist schon erwähnt worden, dass die Gegenkraft bei 2 Volt angelegter Spannung ungefähr 1 Volt beträgt. Man kann leicht ausrechnen, welchen Wert sie in jedem Falle hat, wenn man die angelegte Spannung, die Stromstärke und den wirklichen Widerstand kennt. Ich will hier nur erwähnen, dass bei gut genährten Fröschen Beträge von 2 Volt zu beobachten sind, wenn die Spannung der Batterie 8 Volt und mehr beträgt.

Was das Wesen dieser Kräfte anbetrifft, so haben wir sie zweifellos zu den von Peltier entdeckten sekundär-elektromotorischen Erscheinungen zu rechnen, die später besonders von du Bois-Reymond, Hermann und Hering genauer untersucht worden sind ${ }^{\mathbf{1}}$ ). Mit diesem Namen benennt du Bois-Reymond "elektromotorische Erscheinungen, welche ein fremder Strom in der durchflossenen Strecke selber erzeugt, und", fährt er fort, „die daher meist nur dann erkannt werden, sofern sie den fremden Strom überdauern" ${ }^{2}$ ). Um sie nachzuweisen, bediente sich dieser Forscher der Wippenmethode: das durehströmte Organ wurde plötzlich von der Stromquelle abgetrennt und mit einem Galvanometer verbunden, welches dann durch den Depolarisationsstrom mehr oder minder stark abgelenkt wurde. Ähnlich experimentierten auch Hermann und Hering.

Nun weist schon Herman $\mathbf{n}$ darauf hin, dass man nicht Zustände, die nach der Öffnung beobachtet sind, ohne weiteres auf die Schliessungszeit übertragen kann. Deshalb konnten diese Forseher

1) Die Literatur ist in Biedermann, Elektrophysiologie S. $376 \mathrm{ff}$. angegeben.

2) Arch. f. Physiol. 1884 S. 2 . 
kein ganz treues Bild von den während der Durchströmung bestehenden Gegenkräften bekommen, zumal da die Umschaltung zum Galvanometer immer einen gewissen Zeitverlust bedingt, und da ausserdem, wie Hermann und Hering betonen, die Öffnung des polarisierenden Stromes selbst elektromotorische Kräfte weckt (den Aktionsstrom an der Anode).

Die von mir hier benutzte Methode der gleichzeitigen Widerstandsmessung mit Gleichstrom und Wechselstrom ist diesen Einwänden entrückt. Mit ihr wäre es auch möglich, schon die vou den früheren Untersuchern aufgeworfenen Fragen über die Abhängigkeit der Gegenkraft von der angelegten Spannung, der Stromdichte, der Durchströmungszeit usw. exakt zu entscheiden. Ich habe diese Fragen, die mich zu weit von meiner Aufgabe fortgeführt hätten, nicht weiter verfolgt; wie mir Herr Prof. Gildemeister mitteilt, wird er über Versuche und Überlegungen, die zu diesem Thema gehören, demnächst in diesem Archiv berichten.

Was den Sitz der Kräfte anbetrifft, so ist schon erwähnt worden, dass die Haut die Hauptstelle ibrer Entstehung ist. Aber auch andere Organe, z. B. die Leber und natürlich auch die Muskeln, zeigen auch nach dieser Methode beträchtliche Polarisierbarkeit. Man kann diese Eigensehaft obne besondere Messungen an der mehr oder minder grossen Breite des telephonischen Minimums quantitativ abschätzen. Die Haut übertrifft darin bei weitem alle anderen Organe.

Dabei drängt sich die Frage auf, welcher strukturellen oder sonstigen Eigenschaft die Haut wohl ihre grosse Polarisierbarkeit verdankt. Man könnte da an ihren lamellösen Bau denken. Aber daran allein kann es nicht liegen, denn aufeinandergelegte Schichten von Fliesspapier, Gelatine oder Schweinsblase zeigen keinen wesentlichen Widerstandsunterschied gegenüber Gleich- und Wechselstrom. Auch die zellige Struktur an sich hat mit der Erscheinung nichts zu tun, denn Kartoffeln, Klumpen aus Hefe usw. haben merklich denselben Widerstand, wenn man einmal Wechselstrom, einmal Gleichstrom von 2 Volt Spannung bindurchgehen lässt. Dass feuchte Tonplatten, Wattebäusche, Stärkebrei auch fast gar nicht polarisierbar sind (wenigstens bei den von mir benutzten relativ geringen Spannungen), sei noch der Vollständigkeit wegen erwähnt.

Fast alle Erscheinungen, die ich in dieser Arbeit beschrieben, findet man an metallischen Polarisationsmodellen wieder. Taucht 
man Platinbleche in verdünnte Schwefelsäure, so erweist sich diese Kombination als wenig durchlässig für Gleichstrom, als sehr leicht passierbar für Wechselstrom, und die gleichzeitige Messung mit heiden Stromarten lehrt, dass keine Erböhung des wahren Widerstandes vorhanden ist. Nur scheint hier die Gegenkraft längere Zeit zu brauchen, ehe sie zu voller Grösse entwickelt ist, und sie ist weniger, oder besser ausgedrückt, in anderer Weise von der Vorgeschichte abhängig. Messe ich den scheinbaren Widerstand mit niedriger Spannung, schalte dann einen Versuch mit hoher Spannung ein und gehe dann wieder zu der ersten Spannung zurück, so erweist er sich beim tierischen Präparat jetzt als herabgesetzt, beim Platinmodell aber als unverändert oder gar erhöht.

An walıre metallische Polarisation wird man beim Tier natürlich nicht denken können, sondern im Sinne der modernen Elektrochemie an Konzentrationsveränderungen von Elektrolyten an Grenzflächen. Ich kann meinen Versuchen nichts entnehmen, was mich befähigte, einer der von den verschiedenen Autoren (Nernst, Cremer, $\mathrm{Hab}$ er u. a.) aufgestellten spezielleren Theorien den Vorzug zu geben.

Zum Schlusse ist es mir eine angenehme Pflicht, Herrn Professor Gildemeister für die Anregung zu dieser Arbeit und für die ständige Unterstützung bei der Ausführung derselben meinen besten Dank auszusprechen.

\section{Zusammenfassung.}

In dieser Arbeit ist der elektrische Leitungswiderstand des intakten Froschkörpers mit Gleichstrom und mit Wechselstrom gemessen worden.

Im Einklange mit Tatsachen, die schon lange vom menschlichen Körper bekannt sind, unterscheiden sich beide Widerstände wesentlich voneinander, und zwar ist unter den gleichen Umständen der Gleichstromwiderstand (gemessen mit 2 Volt Spannung) annähernd doppelt so gross als der Wechselstromwiderstand.

Beide Widerstände sind nicht proportional der Grösse und dem Abstand der Elektroden. Sie sind beide, besonders aber der Gleichstromwiderstand, hauptsächlich in der Haut lokalisiert.

Der Gleichstromwiderstand nimmt mit zunehmender Stromstärke ab. 
174 Hermann Galler: Über den elektrischen Leitungswiderstand etc.

Der Wechselstromwiderstand ist in den Grenzen meiner Versuche nicht merklich abhängig von der Stärke und Frequenz der Messströme. Ich kann diese Aussage aber nur unter Vorbehalt machen, da ich nur mit Frequenzen bis zu 1020 pro Sekunde gearbeitet habe, und da das Minimum ziemlich breit und verwaschen war. Dieser Punkt müsste noch mit reinen Sinusströmen, welche mir leider nicht zur Verfügung standen, untersucht werden.

Um festzustellen, ob der Froschkörper wirklich dem Gleichstrom einen höheren Widerstand entgegensetzt als dem Wechselstrom, habe ich gleichzeitige Messungen nach beiden Methoden vorgenommen. Dabei zeigte es sich, dass weder der Wechselstromwiderstand durch den gleichzeitig fliessenden Gleichstrom erhöht wird noch umgekehrt. Der hohe Gleichstromwiderstand wird also nur durch die elektromotorischen Gegenkräfte der Gleichstrompolarisation vorgetäuscht. Die Methode der gleichzeitigen Widerstandsmessung durch Gleichund Wechselstrom mácht es möglich, die Grösse der Polarisation während des Stromdurchganges zu messen. Im Froschkörper können Beträge von 2 Volt auftreten. 